\title{
Il nuovo concorso nazionale italiano di accesso alle scuole di specializzazione: luci e ombre
}

\author{
Carmela Errichiello', Maria Grazia De Gregorio² \\ ${ }^{1}$ Nefrologia e Nefrologia dei Trapianti, Dipartimento Specialità Medico-Chirurgiche, Azienda Ospedaliero-Universitaria Careggi, Firenze \\ ${ }^{2}$ Malattie dell'apparato cardiovascolare, Dipartimento del Cuore e dei Vasi, Azienda Ospedaliero-Universitaria Careggi, Firenze
}

\section{New italian competition for medical specialities: lights and shadows}

A new Italian competition for medical specialities started in 2014. It is an innovative model of selection in Italy that focuses on the global knowledge of participants. This competition gives the chance to choose the favorite speciality but it has some technical and organizative problems.

Keywords: Competition, Young medical doctor, Medical specialities

\section{Introduzione}

L'accesso alla scuola di specializzazione è da sempre una tappa fondamentale per il giovane medico. Le polemiche e, spesso, le ingiustizie legate alle modalità del precedente concorso sono state più volte al centro di accese discussioni, manifestazioni nazionali e, addirittura, vicende giudiziarie. Negli ultimi anni, è, quindi, emersa la necessità di proporre un sistema che premiasse il merito e che valorizzasse la preparazione reale oltre al curriculum vitae. II concorso nazionale è stato una conquista e, anche se presenta molte luci, esistono ancora diverse ombre. A tutti gli aspiranti nefrologi in attesa del concorso di specializzazione è rivolta la nostra personale esperienza in relazione alle "nuove" modalità di concorso e alla riduzione della durata del corso di formazione a 4 anni.

\section{Il percorso formativo}

Siamo specializzande in Nefrologia e in Malattie dell'Apparato Cardiovascolare dell'AOU Careggi di Firenze. Entrambe abbiamo completato gli studi di Medicina e Chirurgia presso I'Università Federico II di Napoli e frequentato, dal $4^{\circ}$ anno del corso di laurea, il reparto e gli ambulatori dell'Unità Operativa Complessa di Nefrologia e Dialisi della medesima Uni-

Accepted: July 2, 2015

Published online: July 20, 2015

Indirizzo per la corrispondenza:

Carmela Errichiello

Nefrologia e Nefrologia dei Trapianti

Dipartimento Specialità Medico-Chirurgiche

Azienda Ospedaliero-Universitaria Careggi, Firenze

carmela_errichiello@virgilio.it versità. Tale esperienza è stata molto formativa per la nostra preparazione e non va, di certo, accantonata. La frequentazione di un reparto come quello di Nefrologia è fondamentale per farsi un'idea sia sulle principali tematiche nefrologiche, dalla dialisi al trapianto e dall'insufficienza renale acuta a quella cronica, sia sull'ampiezza e sulla sovrapposizione con altre discipline che questa branca comprende: dall'istopatologia all'equilibrio acido base e dall'ipertensione arteriosa ai disturbi endocrini.

Questi anni possono essere decisivi per effettuare la giusta scelta in merito alla propria futura specialità e per far nascere dentro di sé quell'interesse e quella passione che sono fondamentali per affrontare al meglio il proprio futuro.

\section{Il concorso}

Abbiamo affrontato il nuovo concorso di specializzazione l'anno scorso nella totale incertezza della preparazione, e, per tale motivo, riteniamo doveroso condividere la nostra personale esperienza con chi oggi si ritrova ad affrontare le stesse paure e speranze.

Il concorso ha avuto una durata massima di 4 giorni per coloro che come noi hanno effettuato le prove in tutte e tre le macroaree (medica, chirurgica e servizi). Per ogni macroarea era possibile scegliere al massimo due specializzazioni. II primo giorno era dedicato alla parte generale, il secondo giorno alla macroarea clinica, il terzo a quella chirurgica e il quarto alla branca dei servizi. Alle domande di macroarea seguivano quelle specifiche per ciascuna scuola di specializzazione.

L'assegnazione delle sedi in cui si sarebbero svolte le prove è avvenuta in base a un criterio puramente anagrafico, assegnando, quindi, ciascun partecipante a una determinata città in base alla sua data di nascita, senza tenere in conside- 
razione l'effettiva distanza dal luogo di residenza. Tale scelta è stata fatta per garantire maggiori trasparenza e imparzialità nello svolgimento delle prove, non tenendo conto del disagio che molti di noi hanno dovuto affrontare per raggiungere tali sedi. Questo ha provocato un dispendio economico, ma, principalmente, un carico emotivo aggiuntivo che, in alcuni casi, ha pesato sul risultato finale.

I quiz erano presentati in formato elettronico in aule informatiche, alle quali si poteva accedere previo riconoscimento tramite documento di identità valido. Una volta sistemati tutti i concorrenti, con modalità diverse da sede a sede, i commissari del ministero provvedevano a fornire una password giornaliera nazionale pochi minuti prima dell'inizio della prova. Il tempo a disposizione per la prova era prestabilito e non era possibile allontanarsi dall'aula in anticipo.

Gli elementi del concorso più controversi sono stati sicuramente il contenuto e la qualità delle domande. II nostro studio si era incentrato principalmente sulle patologie delle diverse specialità che avevamo scelto. In particolare, per la Nefrologia abbiamo integrato lo studio dei libri di testo universitari con le evidenze delle Linee Guida e degli articoli scientifici.

Ci siamo comunque ritrovate a dover rispondere a molte domande su batteriofagi e sui virus a DNA o RNA o, ancora, sulle tappe biochimiche e sui cofattori enzimatici delle vie metaboliche. È stato come ritornare al primo anno di Medicina e Chirurgia e sentirsi considerati ancora studenti e non medici. Purtroppo, questa prova ha penalizzato la maggior parte dei concorrenti, perché tale punteggio è stato sommato a quello dei giorni successivi. È vero che la preparazione di un medico deve essere globale, ma alcune conoscenze di base non dovrebbero essere così tanto valorizzate rispetto al resto.

Il secondo giorno è stato segnato da un errore grossolano da parte di chi avrebbe dovuto garantire la corretta gestione dei quiz, con l'inversione delle prove di area medica con quella dei servizi, scatenando lira di noi giovani medici che ci siamo sentiti beffati e umiliati da un sistema che compie errori così palesi quando in gioco ci sono il futuro e le carriere di dodicimila persone.

Le graduatorie con i relativi scorrimenti sono state un altro punto cruciale del concorso. Per ogni specialità veniva stilata una lista dei partecipanti con i relativi voti delle prove e, se vincitori, era indicata la sede a cui erano stati destinati. L'assegnazione definitiva avveniva solo quando il candidato rientrava nella sua prima scelta di sede o, comunque, nella sede scelta che risultava più in alto nella lista delle preferenze compilata al momento dell'iscrizione. Questo tipo di sistema ha comportato che coloro che non erano stati immediatamente assegnati fossero costretti ad aspettare lo scorrimento delle graduatorie per tempi estremamente lunghi. Chi, inoltre, risultava assegnato in un'unica graduatoria diversa da quella della specializzazione a cui ambiva di più aveva due possibilità: accettare una specializzazione che non era la sua prima scelta per non perdere il posto, decadendo automaticamente dalle altre graduatorie, oppure rinunciare al posto non iscrivendosi e sperando di rientrare con il tempo nella sua prima scelta.

In linea di massima, ci sentiamo di annoverare tra i vantaggi dell'attuale concorso:

1. I'idea di un concorso meritocratico che premi lo studio, l'impegno e il merito, riducendo il peso del curriculum vitae sulla valutazione generale del candidato ed escludendo dal punteggio tutte quelle variabili (pubblicazioni e simili) che possono inficiare l'imparzialità della valutazione nell'ambito delle diverse scuole. Questo permette al candidato di frequentare il reparto per arricchire la propria esperienza personale senza sentire il peso di dover accumulare "punteggio" in qualche modo;

2. la possibilità di scegliere tra più sedi e decidere quelle più adeguate alla propria formazione, indipendentemente da dove è avvenuta la frequentazione negli anni precedenti;

3. la possibilitâ di scegliere una specializzazione indipendentemente dalla frequentazione del reparto e dall'attinenza della tesi;

4. la maggiore oggettività nella valutazione delle prove che, di fatto, sono uguali per tutti.

Tra gli svantaggi vanno segnalati:

1. le modalità di scorrimento delle graduatorie che hanno costretto alcuni concorrenti ad accettare posti in sedi o in specialità diverse dalle loro prime scelte per il timore di restare fuori da qualunque graduatoria;

2. i tempi infiniti di scorrimento che si sono protratti fino a pochi mesi fa, come è possibile costringendo i vincitori a trasferirsi nel giro di pochi giorni in città anche molto distanti, senza un alloggio definitivo;

3. disuguaglianza dal punto di vista temporale dell'inizio dell'attività lavorativa tra gli specializzandi del primo anno a livello nazionale;

4. carenza di informazione grazie alla quale effettuare una scelta consapevole delle sedi universitarie;

5. aumento dei ricorsi anche su base nazionale per interpretazione delle domande e delle modalità degli scorrimenti.

Eventuali punti da migliorare, a nostro parere, sono:

1. diffusione di un'informazione organizzata e aggiornata di tutti i piani formativi degli atenei nazionali, in modo tale da permettere realmente una scelta orientata e consapevole ai concorrenti;

2. assegnazione delle sedi che, a nostro giudizio, dovrebbe rispettare la vicinanza con il luogo di residenza e non abbinare il criterio anagrafico con quello alfabetico delle province;

3. parità nell'assegnazione delle postazioni dei computer tramite sorteggio in tutte le sale; 
4. eliminazione dell'unità centrale dei PC per evitare problemi tecnici che, purtroppo, si sono verificati in varie sale e che hanno ritardato le prove di alcuni concorrenti, costringendo tutti gli altri della stessa sala ad aspettare che quel singolo partecipante finisse in ritardo dopo il riavvio del programma per visualizzare i risultati della giornata;

5. precisione e attinenza delle domande delle prove con revisione oculata dei quiz;

6. velocizzazione del processo di scorrimento delle graduatorie.

\section{Durata delle scuole di specializzazione}

Per quanto concerne la durata della specializzazione, il dibattito è ancora aperto. Abbiamo dovuto affrontare una scelta importante per la nostra formazione e la nostra futura carriera senza una valida informazione sulle differenze dei piani formativi nei 4 o 5 anni. Noi due abbiamo scelto in base alle esigenze personali, alle aspettative e all'organizzazione della propria scuola. C'è chi ha optato per i 4 anni per immettersi prima nel mondo del lavoro e chi, invece, ha scelto 5 anni per fare più tappe o un periodo di formazione esterno con più tranquillità. Dai prossimi anni sarà applicata, senza possibilità di scelta, la riduzione della durata delle scuole di specializzazione. Al momento non esistono dati sufficienti per sapere se questa sia stata o meno una mossa vincente. L'unica cosa che, magari, ci sentiamo di dire è che la riduzione non dovrebbe essere applicata in maniera uniforme a tutte le scuole ma che, al contrario, andrebbe calibrata valutando la necessità di tempo e di pratica di cui lo specializzando ha bisogno per diventare un professionista nella propria disciplina e adeguando l'offerta formativa all'eventuale riduzione.

\section{Conclusioni}

Sebbene esistano ancora molti aspetti da migliorare, il concorso nazionale rappresenta un grosso passo in avanti per la meritocrazia e per la possibilità di poter scegliere direttamente la propria formazione. Gli anni della specializzazione devono essere i più intensi e propositivi della nostra vita lavorativa, perciò bisogna scegliere con cognizione di causa la propria strada e sfruttare al massimo le opportunità.

\section{Disclosures}

Financial support: No financial support was received for this submission. Conflict of interest: The authors have no conflict of interest. 